\title{
Fitting in or opting out: A review of key social-psychological factors influencing a sense of belonging for women in physics
}

\author{
Karyn L. Lewis, ${ }^{1}$ Jane G. Stout, ${ }^{2}$ Steven J. Pollock, ${ }^{3}$ Noah D. Finkelstein, ${ }^{3}$ and Tiffany A. Ito ${ }^{1}$ \\ ${ }^{1}$ Department of Psychology and Neuroscience, University of Colorado Boulder, \\ Boulder, Colorado 80309, USA \\ ${ }^{2}$ Computing Research Association, 1828 L Street, NW, Suite 800, Washington, DC 20036, USA \\ ${ }^{3}$ Department of Physics, University of Colorado Boulder, Boulder, Colorado 80309, USA
}

(Received 30 January 2015; published 1 August 2016)

\begin{abstract}
[This paper is part of the Focused Collection on Gender in Physics.] A number of cultural, social, environmental, and biological factors have been suggested to explain women's relatively lower representation in physics and other science, technology, engineering, and math (STEM) fields. Given its persistence, the causes of gender disparities are likely to be complex and multiply determined. In this review paper, we discuss how a sense of belonging relates to women's interest, persistence, and achievement in physics. We explore what it means to "fit in" and belong in academic contexts, the situational and interpersonal antecedents to belonging, and the consequences of a lack of belonging. We review the empirical evidence for the efficacy of interventions designed to bolster a sense of belonging. Based on these interventions we conclude the paper with a number of practical recommendations to affirm women's sense of belonging and create more welcoming and inclusive physics environments for all students.
\end{abstract}

DOI: 10.1103/PhysRevPhysEducRes.12.020110

\section{INTRODUCTION AND BACKGROUND}

The number of women participating in physics is strikingly low at all levels. In fact, although the number of U.S. women receiving bachelor's degrees in physics showed small but steady increases for the last half of the 20th century, the past decade has seen this growth stagnate and the percentage of women earning Bachelor's degrees hovers at around 20\% [1]. This pattern is true of many other science, technology, engineering, and math (STEM) fields, but not all. In the life sciences such as biology, and the social sciences, the number of women is at parity with if not great than the number of men [2], which highlights the importance of understanding why only some disciplines suffer from gender disparities in accomplishment and retention. This is also imperative given these gender gaps come at a cost both to women who lose out on the lucrative STEM jobs and to our society as a whole because a homogenous STEM workforce hampers scientific progress and technological innovation [3].

Although contextual factors that negatively impact women's performance are well documented [4], the evidence for underlying innate ability differences in quantitative skills is scant [5-7] and even if true could not fully account for the magnitude of extant gender gaps in STEM participation. If differential ability is not to blame, what

Published by the American Physical Society under the terms of the Creative Commons Attribution 3.0 License. Further distribution of this work must maintain attribution to the author(s) and the published article's title, journal citation, and DOI. then can explain women's underrepresentation in physics? Given the pervasiveness and persistence of these gender disparities, the causes are likely to be complex and multiply determined. Ruling out innate ability differences points to sociocultural factors as a key culprit, and many such factors have been suggested to explain women's relatively lower representation in physics and other STEM fields [8-10]. A full treatment of all the potential sociocultural and motivational factors that underlie gender gaps in STEM representation is beyond the scope of this article. Instead, here we hone in on one important social psychological process - students' sense of belonging within the academic environment. We define academic belonging as the extent to which individuals feel like a valued, accepted, and legitimate member in their academic domain. Belonging has long been recognized as an innate human need and an important driver of physical and psychological well being [11-13]. Because academic endeavors are not pursued in isolation but rather are replete with complex social interactions and relationships, we believe it is important to acknowledge and understand the role of belonging in academic performance and persistence.

We begin by reviewing research findings that highlight how the need to feel a sense of belonging in physics could act as a core psychological process through which multiple factors operate to encourage the participation of men while inhibiting the participation of women. We then highlight other research that demonstrates what can be done to boost students' sense of belonging and potentially encourage the participation of women and other underrepresented groups 
in physics and other STEM fields. We primarily restrict our review to empirical findings conducted in higher education settings given that research suggests that STEM attrition increases at upper stages in the academic hierarchy [14]. Moreover, the choices of higher education students are more freely made than those of $\mathrm{K}-12$ students (where curriculum requirements constrain choices). Because student choice plays a large role in undergraduate STEM experiences (particularly in the first two years), sense of belonging in college STEM must be a critical area of focus.

\section{SENSE OF BELONGING IN ACADEMIC DOMAINS}

Why should it matter whether or not students feel a sense of belonging in their academic domain? The human need to belong is among the most powerful motivators of social behavior [11]. Theorists suggest that this need is universal and innate given that strong social ties were critical to the success of our species and we therefore evolved psychological mechanisms that lead us to seek out and maintain close personal relationships with others [11]. Because this need is so ingrained, we constantly monitor our belonging status and can detect even very subtle indications (e.g., lack of eye contact [15]) that our belonging is threatened [16]. When people perceive threats to their belonging (e.g., when they feel excluded or rejected), they show strong adverse reactions and their functioning can be negatively affected in multiple ways. For instance, after experiencing exclusion, people show poorer self-regulation [17], more self-defeating behavior [18], and even poorer cognitive performance [19]. To relieve the psychologically adverse experience of thwarted belonging, people typically act quickly to try to restore threatened social ties and/or create new ones [20].

Research and theory regarding belonging needs initially developed in the context of studying close interpersonal relationships, but we now know that the importance of belonging cuts across all aspects of daily life. Most relevant to this paper, sense of belonging has been receiving increasing attention in models of academic achievement and persistence, where it has been indicated as a determinate of success and retention [21]. In the current paper, we define academic belonging as the extent to which students subjectively perceive that they are valued, accepted, and legitimate members in their academic domain. Depending on the field of inquiry, this same or similar psychological experience can also be referred to as "sense of school membership" or "sense of community." We believe that, in essence, these terms and concepts all refer to the same psychological experience of sense of belonging and this is the term we use throughout the paper. A key consideration when measuring academic belonging is the level of specificity in question (e.g., belonging to the university, to a particular domain, or to a particular class); we take care to attend to the level of specificity under consideration in the research we review below.
We differentiate sense of belonging from the related psychological experience of identification. Identification refers to the degree to which a particular domain is an important element of one's self-definition (e.g., "I'm a math person") [22]. Whereas belonging reflects one's perceived fit within a group or entity, identification is instead the importance one places on being a member of that group or entity. Belonging and identification have occasionally been lumped together in definition and/or in measurement [23], but we see utility in differentiating these processes, particularly when considering the experience of marginalized groups for whom it seems possible to be highly identified while simultaneously low in sense of belonging. Furthermore, although belonging and identification are often positively correlated with one another, when the relative contributions of identification and sense of belonging in predicting academic outcomes are considered, sense of belonging tends to be more strongly linked to academic persistence [24].

\section{A. The importance of sense of belonging for attitudes and academic success}

Not surprisingly, research has shown that higher academic belonging is associated with generally more positive affective experiences; students who report feeling more connected with their school community also report liking school more [25]. But sense of belonging has also been linked with outcomes directly related to achievement. For instance, Zumbrunn and colleagues [26] investigated college students' sense of belonging to their university in relation to two important components of academic achievement: self-efficacy (beliefs about the extent to which the self is capable of successfully completing tasks [27]) and task utility (perceiving a task or activity to be important, useful, and worthwhile [28]). They found that students who felt more belonging within their university also reported higher self-efficacy for course tasks and assignments and also reported seeing more usefulness and value in their courses. Similarly, Pittman and Richmond [29] found that sense of belonging at the university level predicted perceived academic competence; students who felt more belonging with their university felt more competent in their scholastic pursuits. Importantly, this study also showed that students who reported a higher sense of belonging were not only more motivated than peers low in belonging, they also reported higher grades. Another study investigating sense of belonging at the university level linked sense of belonging with students' intentions to persist at the university [30]. Even after controlling for other known correlates of persistence (e.g., financial difficulties, standardized test performance), students in this study who reported higher sense of belonging also reported stronger intentions to persist at the university.

The above studies draw on theoretical models that specify sense of belonging as a causal factor in academic 
outcomes, but the correlational nature of these results means causal relationships are hinted at but not confirmed. However, there is experimental research that directly indicates the causal role of belonging in academic outcomes. Walton and colleagues used an experimental manipulation to induce a sense of belonging in some participants and compared their outcomes to those for control participants [31]. Although the conditions used to induce belonging were minimal (in one study participants were led to believe they shared a birthday with another student in a particular academic field, in another study participants shared music preferences with a peer), the effects of these manipulations were significant: participants in the belonging condition reported more motivation for and even persisted longer on an unsolvable math puzzle.

To summarize, the research reviewed above indicates that students function better in contexts where they feel a strong sense of fit and their need to experience a sense of belonging is satisfied. Correlational evidence shows that students high in sense of belonging are more motivated, more engaged, and even show better performance and higher intentions to persist. The causal link hinted at in these studies whereby sense of belonging directly leads to better academic outcomes is further supported by experimental work showing even under very minimal conditions, feeling a sense of belonging with other students increases motivation and performance.

\section{B. The importance of domain-specific belonging}

In addition to measuring belonging at the level of the university, or with academic peers in general, belonging can also be measured at the level of a specific discipline. Just as general school and academic belonging predicts general academic success, domain-specific belonging predicts domain-specific outcomes. For instance, Freeman and colleagues [32] examined sense of belonging in a specific college class and tested whether sense of class belonging would predict self-efficacy, task utility, and intrinsic motivation (engaging in activities for the sake of personal interest or enjoyment [33]). They found a similar pattern of correlations as those reviewed above-sense of belonging in the class was positively associated with each of these academic outcomes; students who felt more belonging in class were more likely to report high self-efficacy in that class, high motivation for completing class activities and tasks, and perceived the class to be more useful and valuable. Similarly, Wilson and colleagues measured sense of belonging at varying levels of specificity-belonging in a particular class, belonging in the major, and belonging in the university-and found that students' self-reported academic engagement for their classes and work in their major correlated most strongly with belonging assessed at the more specific levels (belonging in a class and the major) [34].
Domain-specific belonging is also related to domainspecific persistence. For instance, London and colleagues found that women who reported lower sense of belonging specifically in their STEM major were more likely to expect to switch out of their major [35]. Qualitative research also supports this link. Specifically, Seymour and Hewitt conducted an extensive ethnographic investigation of why undergraduates leave science and their interviews with women who left STEM majors suggested a common reason for women's attrition was feeling like outsiders in the traditionally male-centered culture in STEM [36]. Seymour and Hewitt's work suggest this stems from the isolation that comes with having few same-sex peers, but also from the traditional "weed-out system" characteristic of many STEM fields that encourages behavior (e.g., assertiveness, self-promotion, competitiveness) that is often at odds with traditional female gender roles [36].

Thus, for physics educators interested in best practices for classroom outcomes, attending to students' subjective feelings of fit and acceptance is an important consideration for maximizing physics performance and retention. Unfortunately, converging evidence suggests that in STEM settings such as physics, women are more likely to experience threats to their belonging $[24,37,38]$. This can start a cascading sequence that leads to poorer outcomes: because belonging is such fundamental need, we constantly monitor our belonging status [39]; when belonging is low, cues relevant to belonging have a greater impact than when belonging is high [39-42]. Taken together, this suggests that a subjective sense of belonging might be more impactful for women in STEM than for men and low belonging is an important potential mechanism for women's decreased representation and achievement in physics. In other words, when men in physics question "do I belong here?", they encounter a resounding "yes!" from an environment replete with other men and positive stereotypes about their group; however, when women question whether they belong, their belonging is instead threatened by a lack of other women and negative stereotypes regarding women's intellectual abilities.

Consistent with this reasoning, when looking specifically at sense of belonging within STEM fields such as physics we have found evidence that belonging is indeed more impactful for women than men [38]. In this work, we assessed academic belonging at the level of the specific domain, and we found that in multiple samples including undergraduate as well as graduate students, women report lower belonging with their STEM field (i.e., computing or physics) than men. These data also show that for women (but not men) belonging was strongly linked to intentions to persist in a cross-sectional sample. Furthermore, in a longitudinal sample belonging was linked to actual persistence for women but not men. Women who had low belonging reported lower intentions to persist and also less 
actual persistence, even after controlling for objective course performance. In contrast, men reported more belonging in the field overall, but belonging was not as strongly linked to persistence as for women, or it did not predict persistence at all.

These results are particularly disconcerting because low belonging not only makes women more pessimistic about their potential in the present moment, but may also affect the way future ambiguous events are viewed and their subsequent impact [37]. In other words, low belonging can potentially lead to a negative feedback loop where once a woman's belonging is called into question (e.g., after an unfriendly exchange with a classmate or receiving a disappointing grade), she becomes more vigilant in looking for further evidence about whether she belongs while also tending to interpret the meaning of any ambiguous cues with a more negative slant leading her to feel even lower belonging [43].

\section{FACTORS THAT AFFECT SENSE OF BELONGING}

The accumulating evidence linking sense of belonging with academic outcomes makes it clear that academic success is not solely an individual process driven by differences in abilities and aptitude. Rather, academic success is also a social process influenced by the extent to which students feel a sense of belonging in their academic environment. We next turn to the question of what factors diminish versus bolster sense of belonging with the goal of outlining specific ways to structure learning environments in order to maximize every students' sense of belonging. These factors include (i) quantity and qualities of peers and role models, (ii) stereotypes about the attributes of people who succeed in STEM, (iii) direct interventions within STEM settings, and (iv) influences from outside the academic environment. We focus on each of these factors in turn in the following subsections.

\section{A. Quantity and qualities of peers and role models}

When trying to determine whether one belongs in a setting, an efficient approach is for people to assess how similar they are to that setting's inhabitants. It is easy to imagine then that seeing very few same-gender peers and role models leads women to naturally conclude "People like me do not belong here." Accordingly, the typical gender imbalance women encounter in STEM settings (e.g., physics) where only about 1 in 5 people are women [2] may be a very blatant cue about who does and does not belong. Dasgupta theorizes that in achievement contexts contact with in-group peers and experts functions as a kind of psychological vaccine that protects people from the negative effects of identity threat and serves to boost sense of belonging [44]. This contact may be especially important for people who are members of marginalized groups who must cope with negative stereotypes about their group's abilities and/or with being a minority in the numerical sense. Consistent with Dasgupta's model, Stout and colleagues have shown that interacting with female peers and having access to female instructors is beneficial for women's identification with math [45].

Is there any evidence to link lack of female peers and role models specifically to women's low sense of belonging in STEM? Murphy and colleagues [46] tested this hypothesis in an experiment where advanced undergraduate students from a range of STEM majors were exposed to a video depicting an ostensible STEM student leadership conference where the gender composition of conference participants was manipulated. When women were exposed to typical asymmetric STEM gender ratios (i.e., women were far outnumbered by men in the video), they reported lower belonging and lower interest in the conference relative to women who were exposed to balanced gender ratios. Furthermore, women also showed greater stress responses when viewing the asymmetric gender ratio video. In contrast, men's belonging, interest, and stress responses were unaffected by the gender representation in the videos.

Although these results suggest that the presence of female peers and role models are beneficial to women, other research suggests that qualities of that person also matter. Looking specifically at the field of computer science, Cheryan and colleagues [47] studied whether role-model gender matters in fostering women's sense of belonging and subsequently their interest in pursuing computer science. Importantly, this study also manipulated whether or not the role-model seemed to fit typical computer science stereotypes (e.g., socially awkward, fanatical about computers and technology). As predicted, Cheryan et al. found that women's sense of belonging did differ depending on qualities of the role model. However, it was not gender that mattered, but rather it was whether or not the role model projected computer science stereotypes. Women's sense of belonging was the lowest when they interacted with a stereotypical role model, regardless of whether that person was a man or a woman, and this interaction in turn was linked to decreased interest in pursuing computer science in the future.

Cheryan and colleagues [48] have also shown that stereotypes about the qualities of STEM inhabitants can be transmitted subtly by the physical environments students encounter in these fields. Participants in this study reported their belonging with computer science and their interest in pursuing this field. They made these evaluations either in an environment that contained physical objects consistent with computer science stereotypes (e.g., a Star Trek poster, computer parts, technical programming books) or in an environment devoid of stereotypical objects and instead filled with neutral items (e.g., nature posters, general interest books). Even though the stereotypic objects were seemingly innocuous, women reported less belonging and 
less interest in computer science when exposed to the stereotypic environment relative to women who encountered a neutral physical environment. In contrast, men's belonging with and interest for computer science did not differ between physical environments. Importantly, this divergence in women's interest for computer science between environments portrayed as stereotypical or not persisted even when the members of that environment were all women themselves. Thus, consistent with the work reviewed above suggesting that it is not role-model gender that matters as much as whether that person embodies stereotypes, these results suggest that even when an environment is replete with women, if that environment is perceived to embody stereotypes, this is problematic for women considering entering the field and has negative consequences for their sense of belonging. Similarly, Chen and Hamilton showed that perceptions of diversity are not only driven by objective inclusivity (i.e., actual numbers of minorities present in a setting) but also by psychological inclusivity (i.e., whether minorities perceive people in a setting to be socially accepting) [49]. All told, this suggests it may not be enough simply to populate STEM environments with more women. The culture of these environments needs to also shift to be openly and obviously accepting of women.

The exact mechanism linking stereotypes and decreased sense of belonging is unclear at this point, particularly given that stereotypes include both negative (e.g., socially inept, loner) and positive (e.g., smart, focused, tech whiz) traits that members of the field are expected to have. At this point, the evidence suggests that exposure to stereotypical cues can highlight a sense of personal incompatibility with the domain, leading women to conclude that in order to participate in that particular field they would have to be different from who they are or want to be and this serves to depress their sense of belonging and dampen their interest in the field.

\section{B. Ability stereotypes}

Unfortunately, the "geeky" scientist stereotype is not the only stereotype with which women in STEM settings must contend. Cultural stereotypes suggesting women's quantitative abilities are inferior to men's are still prevalent and affect women in myriad ways, ranging from depressing their objective performance [50] to negatively impacting their attitudes and identification [51]. Specifically, Steele's seminal research on stereotype threat shows that when a social identity associated with negative stereotypes is made salient, poorer test performance in the stereotyped domain follows [52]. Similar work shows that being outnumbered (i.e., the only woman in a group of men) can also lead women to underperform on math tasks $[53,54]$. This important line of work has highlighted how negative stereotypes can undermine women's objective performance. Given their pernicious nature, it seems likely that the influence of stereotypes extends beyond hurting performance and may affect women in other ways as well. Indeed, new evidence suggests that negative stereotypes regarding women's intellectual abilities can also have detrimental effects on women's sense of belonging in STEM. Good and colleagues [24] assessed women's sense of belonging specific to math. Consistent with past research, they found that sense of belonging in math predicted intentions to persist in math in the future. They also studied what factors might affect sense of belonging in math and found that for women, but not men, sense of belonging was eroded under a particular set of circumstances: Women reported the lowest belonging when they perceived that others in their math environment (e.g., other students, instructors) believed stereotypes that women are less talented than men in math while also perceiving that others believe math is a fixed ability (as opposed to a malleable skill that can be improved over time [55]). Although this research was conducted with students in math fields specifically it seems reasonable to expect these results would apply to physics and other STEM fields, given these fields share the feature of being quantitatively intensive. Indeed, recent work suggests the fields where women are the most underrepresented are also the ones where practitioners generally believe success is dependent on innate natural talent (as opposed to hard work) [56].

Another way ability stereotypes can negatively impact women's sense of belonging is by skewing women's perceptions of the typical challenges people encounter in STEM settings. Smith and colleagues [57] found that compared to men, women in STEM fields reported feeling as though they had to put forth more effort to succeed and were less naturally talented than other students-in other words, women felt less abled than their peers but men did not. Furthermore, these effort concerns (i.e., feeling that one has to work harder than others) were negatively correlated with sense of belonging for women (but not men); women who felt they had to put in more effort to succeed also felt less of a sense of belonging in their field, even when controlling for objective performance. A followup experiment that manipulated whether a novel field was portrayed as male dominated or gender balanced showed that these effort concerns are triggered for women when the field in question is male dominated; women's anticipated effort and belonging within a gender-balanced field did not differ from men's, but women felt as though they would have to put in more effort than peers and reported less anticipated belonging with a field that was perceived as male dominated [57]. Fortunately, it seems possible to counteract this. In another follow-up experiment, Smith and colleagues showed that women who were made to believe that everyone in the male-dominated field had to work hard and put forth a high amount of effort to succeed reported more belonging with that field and higher interest in it relative to women in a control condition where effort was not normalized [57]. 


\section{Direct interventions}

The results reviewed above suggest that being a part of a group that is recognizably different from the majority and encountering stereotypes serve to stifle women's belonging with STEM fields. In light of these results and with the awareness that gender imbalances and stereotypes are unlikely to be completely eradicated any time soon, is there any evidence for interventions that can foster women's belonging in STEM environments? Walton and Cohen suggest that "social belonging may constitute a psychological lever where targeted intervention could yield broad benefits" [58] (p. 1447). In order to pull this "lever", Walton and colleagues $[37,42,58]$ have designed an intervention that targets students' concerns regarding their belonging status. In this intervention students are presented with the ostensible results of a survey of advanced students at their university. These results include quantitative summaries and direct quotes that converge on the main take-away message of the intervention: worrying about belonging is normal early on in college and these concerns tend to lessen over time. Thus this intervention normalizes the experience of feeling a lack of belonging and makes it clear that these concerns are short-lived and can be overcome for most people.

Two studies point to the efficacy of this intervention. First, Walton and Cohen [42] showed that black college students who received this belonging intervention (relative to black students who did not receive the intervention) showed a range of positive outcomes including higher GPAs and more academic engagement. The long term effects of this simple intervention are striking - even 3 years following the brief intervention, black students who received it continued to show more positive outcomes in terms of their academic achievement (i.e., their GPA was higher), but they even showed health benefits and reported higher psychological well being [58]. Most recently, Walton and colleagues tested the belonging intervention with women in engineering and showed similar positive effects [37]. Consistent with the previously reviewed results, the gender gap in engineering achievement was closed for women who received the belonging intervention; the average GPA of women in the intervention group did not differ from men and was significantly higher than women in the control group. Additionally, the belonging intervention seems to have other benefits: women who received the intervention had higher and more stable self-esteem, reported higher confidence in handling stressors, reported more positive experiences in engineering, were more positive about their future potential in engineering, and even reported more male friends within their engineering major.

Across both belonging intervention studies the effects of this intervention were only present for members of stigmatized groups: White students' outcomes were not positively affected by the intervention in the first study [42] and men's outcomes were not affected in the second study
[37]. What is more, the effects of the intervention on women in the second study were most notable in the specific engineering fields that were the most male dominated (i.e., majors were less than $20 \%$ women) and where women presumably experienced the greatest threats to their sense of belonging.

\section{Outside influences}

All the work highlighted in the previous sections relates to aspects within STEM environments that can boost or suppress women's sense of belonging. We now turn to influences on sense of belonging that come from outside the academic environment. Women who persist in STEM must be able to successfully cope with continual threats to belonging and other stressors. Research on coping with stress suggests that social support from close others is one of the most important coping resources [59]. Is there any research linking social support from these outside sources to students' sense of belonging in academic contexts? London and colleagues [35] sampled women starting their first year in college that had selected a STEM major. They tracked women daily for a short duration to assess what factors would be linked to sense of academic belonging in their major. They found that the women reported higher belonging on the days that they also reported higher levels of support from close others (i.e., parents, siblings, close friends). Thus, social support outside the classroom may have positive effects for women in STEM by helping bolster their sense of belonging and buffer women from psychological adversity.

One social psychological intervention that may help remind students of the social ties and resources available to them outside the classroom involves a simple exercise where participants write about important personally held values. This "values-affirmation intervention" has a wide range of positive effects in academic settings [60]. A mechanism through which the values-affirmation intervention seems to procure benefits is by bolstering sense of belonging. Cook and colleagues suggest that the benefit of affirming one's important values comes from making belonging less conditional on environmental forces (e.g., negative feedback from professors and interactions with peers) [61]. Consistent with this explanation, relative to minority students who did not receive the intervention, affirmed minority middle school students' sense of academic belonging was more stable and was less linked with their academic performance. Shnabel and colleagues also found evidence to support this affirmation-belonging link: female college students showed higher math performance following a values-affirmation exercise that specifically targeted belonging themes relative to nonaffirmed women or women who wrote about values that did not highlight social ties [62]. Thus, the values-affirmation intervention may be beneficial because it boosts academic belonging indirectly by bolstering students' social connections outside the classroom. 
The benefits of values affirmation seem to be particularly strong for students from marginalized groups and this exercise helps them cope with the chronic stressor that comes with being a member of a stigmatized group [63]. Specific to women's experiences in STEM settings, Miyake and colleagues have shown that a values-affirmation intervention conducted with women in an introductory physics course served to reduce the typical gender gap in exam performance in that course [64]. The benefits of the affirmation intervention were the most marked for women who tended to believe stereotypes that men are naturally better at physics and thus presumably experienced the most stress in their classroom experiences. Similarly, Walton and colleagues showed that a values-affirmation intervention positively affected the GPA and attitudes of women in engineering, but particularly so for women in the engineering majors that were especially male dominated (i.e., less than $20 \%$ women) [37].

To summarize, women's academic belonging can be impacted by subtle and not so subtle factors ranging from social support outside the classroom to the presence of other women and role models and peers in the classroom that seem to buck social stereotypes and presumably are perceived as more similar to the self. Specific interventions that can positively impact women's academic belonging include brief writing exercises where women bring to mind important personally held values, receiving messages from other students that normalize belonging concerns as a typical part of being in a field, and receiving feedback that normalizes effort and hard work as required for everyone in the field.

\section{RECOMMENDATIONS FOR PHYSICS EDUCATORS}

We conclude here with a list of specific recommendations based on the empirical evidence reviewed above. Importantly, it is often the subjective perception of social support (as opposed to the objective presence of such support) that matters for well being [65]. Thus, although the "first-choice" intervention to reach parity in gender representation in physics would most likely be a very effective route to providing women with objective indicators of their belonging in the field, such a goal is distal at this time given that the number of women in the field remains far below parity. Fortunately, we know that it is possible to intervene on women's subjective perceptions of their belonging status and we end here with five empirically supported recommendations meant to bolster women's sense of belonging in physics.

\section{A. Identify and temper cues that perpetuate the "geeky" scientist stereotype}

Although there are many aspects of the academic environment educators have no control over, educators should avoid symbolic objects (e.g., characters from science fiction) that appeal to only some students, or at least strike a balance by also including physical objects that buck stereotypes or are stereotype neutral (e.g., photos of prominent female physicists, neutral art prints). Educators should also consider the stereotypes that can be communicated via the material they draw upon in lectures, worked examples, and problem sets (e.g., avoid examples that assume knowledge of stereotypical subjects, such as athletics $[66,67])$. At a personal level, educators could also emphasize aspects of their own personality and hobbies that do not fit stereotypes (e.g., reading fiction, visiting museums, crafts).

\section{B. Openly endorse effort and hard work over brilliance}

Emphasizing that math and science skills are not fixed, but instead expandable with effort and hard work, might neutralize the negative ability stereotypes women encounter in STEM settings. To do this, educators should strive to praise effort and hard work over natural talent, point out famous scientists' failures, be open about one's own struggles and challenges, and acknowledge that science is difficult and requires effort, determination, and hard work from everyone. In addition to being frank about one's own experiences at a personal level, at a structural level educators should also enact classroom practices that connote the importance of effort, hard work, and growth. Specifically, instead of using grading systems that are heavily based on high-stakes tests performance, consider rewarding students for seeking out critique, revising work accordingly, and showing improvement over time.

\section{Send messages that concerns about belonging are normal and fade with time}

Physics educators and other successful role models (e.g., TAs) should consider sharing their own personal concerns with belonging and how they have coped with these worries. Student groups can also be key in transmitting these messages. Physics educators should facilitate and support student organizations (e.g., Society of Women in Physics) that allow outlets for students to interact informally and share their concerns about belonging and even more importantly hear from others who have coped with the same struggles.

\section{Consider the social context constructed in the classroom}

Physics educators should structure classroom practices in ways that maximize students' opportunities to create positive social connections. One way this might be achieved is by emphasizing interactive practices and student-centered approaches where students are engaging with one another more frequently compared to traditional instructor-centered methods. For instance, in the Jigsaw Classroom [68] model instructors place students in groups that are heterogeneous with respect to abilities and demographic characteristics (although women should not be 
isolated in token roles). Each individual in the group learns a unique segment of a task or problem so that the group members are dependent on one another to successfully complete the task or solve the problem. This kind of cooperative approach may benefit women in particular because the active participation required of each group member means women cannot be relegated to peripheral support positions (e.g., note taker) as they often are in group work [69]. In addition, this model also affords opportunities for women to observe other students struggle and make mistakes which may serve to normalize encountering difficulties. To the degree that women's perceptions that classmates endorse natural ability views of STEM achievement negatively affects their own belonging, such equal status contact in which hard work is normalized could dispel perceptions that classmates expect STEM achievement to result from natural talent more so than hard work. Working in groups may also benefit women because connecting with and getting to know one's classmates at a deeper level highlights the variability in peers' personality traits and abilities, which could help dispel stereotypes.

\section{E. Consider the broader social context students are a part of outside the classroom}

Outside social support buffers vulnerable students' sense of belonging from threats within the academic environment. Although physics educators cannot control students' social environments outside the classroom, research on the values-affirmation intervention suggests that including this brief exercise in course curriculum reminds students of their outside social resources and can in turn benefit women by boosting their sense of belonging. Another important way to be aware of and connect with students' broader social contexts is to relate abstract subject matter to students' lives outside the classroom. This can be achieved by helping students understand the relevance of what they are learning to their daily lives [70] and by highlighting the concrete ways science benefits society [71].

\section{CONCLUSION}

The evidence reviewed here highlights the importance of belonging in academic pursuits. We have focused on the role belonging can play in women's pursuit of physics in particular, but it is important to note that women are not the only underrepresented group in these contexts. Some of the same processes we explore in this paper (i.e., the role of negative ability stereotypes, being a member of a group that is obviously different from the majority) may apply to racial minorities as well, but there are also likely unique issues related to how racial minorities experience academic belonging require further exploration. It is also important to recognize that women are not a single entity. Dichotomizing gender to compare women as a whole to their male counterparts is problematic because gender identity intersects with other identities in important ways $[72,73]$. Unfortunately, however, sample sizes rarely allow for a complete examination of the differential experiences of people who simultaneously belong to multiple stigmatized groups (e.g., women who are Latina, black, firstgeneration college students, and/or LGBTQ) and this is certainly true of the studies reviewed here. In this paper we have attempted to summarize the experiences of women, but this comes at the potential cost of overgeneralizing the experiences of the most dominant (i.e., middle-class white women) onto those at the intersections.

While additional work is needed to examine the degree to which the results here are moderated by other factors, the research to date consistently suggests that, on average, women do not feel as they fit and are accepted in STEM, and are more likely to opt out than men. Thus, we believe a reliable route to increased representation of women in physics is to narrow the gap between women's and men's perceptions of belonging and create inclusive environments that affirm women's belonging just as much as men's. It is our hope that this paper underlines the importance of attending to the aspects of academic contexts that can connote belonging and arms educators with strategies to work to modify the environment students encounter in order to foster the belonging of all students.

\section{ACKNOWLEDGMENTS}

This work was funded in part by National Science Foundation Grant No. HRD-1251590.
[1] P. H. Mulvey and S. Nicholson, Physics Bachelor's Degrees: Results from the 2010 Survey of Enrollments and Degrees, Statistical Research Center of the American Institute of Physics, 2012.

[2] National Science Foundation, National Center for Science and Engineering Statistics, Women, Minorities, and Persons with Disabilities in Science and Engineering: 2013.
Special Report NSF 13-304. Arlington, VA, http://www .nsf.gov/statistics/wmpd/.

[3] National Academy of Sciences, National Academy of Engineering, and Institute of Medicine of the National Academies, Rising above the Gathering Storm: Energizing and Employing America for a Brighter Economic Future (National Academies Press, Washington, DC, 2007). 
[4] S. J. Spencer, C. M. Steele, and D. M. Quinn, Stereotype threat and women's math performance, J. Exp. Soc. Psychol. 35, 4 (1999).

[5] S. M. Lindberg, J. S. Hyde, J. L. Petersen, and M. C. Linn, New trends in gender and mathematics performance: A meta-analysis, Psychol. Bull. 136, 1123 (2010).

[6] N. M. Else-Quest, J. S. Hyde, and M. C. Linn, Crossnational patterns of gender differences in mathematics: A meta-analysis, Psychol. Bull. 136, 103 (2010).

[7] E. A. Spelke, Sex differences in intrinsic aptitude for mathematics and science?: A critical review, Am. Psychol. 60, 950 (2005).

[8] S. J. Ceci, W. M. Williams, and S. M. Barnett, Women's underrepresentation in science: Sociocultural and biological considerations, Psychol. Bull. 135, 218 (2009).

[9] National Academy of Sciences, Beyond Bias and Barriers: Fulfilling the Potential of Women in Academic Science and Engineering (National Academic Press, Washington, DC, 2006).

[10] D. F. Halpern, C. P. Benbow, D. C. Geary, R. C. Gur, J. S. Hyde, and M. A. Gernsbacher, The science of sex differences in science and mathematics, Psychol. Sci. Publ. Interest 8, 1 (2007).

[11] R. F. Baumeister and M. R. Leary, The need to belong: Desire for interpersonal attachments as a fundamental human motivation, Psychol. Bull. 117, 497 (1995).

[12] A. H. Maslow and R. Lowry, Toward a Psychology of Being (Van Nostrand Reinhold, New York, 1968).

[13] C. L. Hawkley and J. T. Cacioppo, Loneliness matters: A theoretical and empirical review of consequences and mechanisms, Ann. Behav. Med. 40, 218 (2010).

[14] S. Hurtado, K. Eagan, and M. Chang, Degrees of success: Bachelor's degree completion rates among initial STEM majors, Higher Education Research Institute, University of California, Los Angeles, http://www.heri.ucla.edu/nih/ downloads/2010\%20-\%20Hurtado,\%20Eagan,\%20Chang $\% 20-\% 20$ Degrees $\% 20$ of\%20Success.pdf.

[15] E. D. Wesselmann, F. D. Cardoso, S. Slater, and K. D. Williams, To be looked at as though air: Civil attention matters, Psychol. Sci. 23, 166 (2012).

[16] N. L. Kerr and J. M. Levine, The detection of social exclusion: Evolution and beyond, Group Dynam. 12, 39 (2008).

[17] R. F. Baumeister, C. N. DeWall, N. J. Ciarocco, and J. M. Twenge, Social exclusion impairs self-regulation, J. Pers. Soc. Psychol. 88, 589 (2005).

[18] J. M. Twenge, K. R. Catanese, and R. F. Baumeister, Social exclusion causes self-defeating behavior, J. Pers. Soc. Psychol. 83, 606 (2002).

[19] R. F. Baumeister, J. M. Twenge, and C. K. Nuss, Effects of social exclusion on cognitive processes: Anticipated aloneness reduces intelligent thought, J. Pers. Soc. Psychol. 83, 817 (2002).

[20] C. N. DeWall and B. J. Bushman, Social acceptance and rejection the sweet and the bitter, Curr. Dir. Psychol. Sci. 20, 256 (2011).

[21] C. Goodenow, Classroom belonging among early adolescent students relationships to motivation and achievement, J. Early Adolesc. 13, 21 (1993).
[22] J. L. Smith and P. H. White, Development of the domain identification measure: A tool for investigating stereotype threat effects, Educ. Psychol. Meas. 61, 1040 (2001).

[23] K. E. Voelkl, Measuring students' identification with school, Educ. Psychol. Meas. 56, 760 (1996).

[24] C. Good, A. Rattan, and C. S. Dweck, Why do women opt out? Sense of belonging and women's representation in mathematics, J. Pers. Soc. Psychol. 102, 700 (2012).

[25] D. Solomon, V. Battistich, D.-I. Kim, and M. Watson, Teacher practices associated with students' sense of the classroom as a community, Soc. Psychol. Educ. 1, 235 (1996).

[26] S. Zumbrunn, C. McKim, E. Buhs, and L. R. Hawley, Support, belonging, motivation, and engagement in the college classroom: a mixed method study, Instrumentation Science and Technology 42, 661 (2014).

[27] A. Bandura, Self-efficacy mechanism in human agency, Am. Psychol. 37, 122 (1982).

[28] J. S. Eccles and W. Allan, In the mind of the actor: The structure of adolescents' achievement task values and expectancy-related beliefs, Pers. Soc. Psychol. Bull. 21, 215 (1995).

[29] L. D. Pittman and A. Richmond, Academic and psychological functioning in late adolescence: The importance of school belonging, J. Exp. Educ. 75, 270 (2007).

[30] L. R. M. Hausmann, J. W. Schofield, and R. L. Woods, Sense of belonging as a predictor of intentions to persist among African American and white first-year college students, Res. High. Educ. 48, 803 (2007).

[31] G. M. Walton, G. L. Cohen, D. Cwir, and S. J. Spencer, Mere belonging: The power of social connections, J. Pers. Soc. Psychol. 102, 513 (2012).

[32] T. M. Freeman, L. H. Anderman, and J. M. Jensen, Sense of belonging in college freshmen at the classroom and campus levels, J. Exp. Educ. 75, 203 (2007).

[33] R. M. Ryan and E. L. Deci, Self-determination theory and the facilitation of intrinsic motivation, social development, and well-being, Am. Psychol. 55, 68 (2000).

[34] D. Wilson, D. Jones, F. Bocell, J. Crawford, M. J. Kim, N. Veilleux, T. Floyd-Smith, R. Bates, and M. Plett, Belonging and academic engagement among undergraduate STEM students: A multi-institutional study, Res. High. Educ. 56, 750 (2000).

[35] B. London, L. Rosenthal, S. R. Levy, and M. Lobel, The influences of perceived identity compatibility and social support on women in nontraditional fields during the college transition, Basic Appl. Soc. Psychol. 33, 304 (2011).

[36] E. Seymour and N. M. Hewitt, Talking about Leaving: Why Undergraduates Leave the Sciences (Westview Press, Boulder, CO, 1997).

[37] G. M. Walton, C. Logel, J. M. Peach, S. J. Spencer, and M. P. Zanna, Two brief interventions to mitigate a "chilly climate" transform women's experience, relationships, and achievement in engineering, J. Educ. Psychol. 107, 468 (2011).

[38] K. L. Lewis, J. G. Stout, G. L. Cohen, S. J. Pollock, N. D. Finkelstein, and T. A. Ito, Fitting in to move forward: using a belonging framework to understand gender disparities in persistence in the physical sciences, technology, engineering, and mathematics (pSTEM) (to be published). 
[39] W. L. Gardner, C. L. Pickett, and M. B. Brewer, Social exclusion and selective memory: How the need to belong influences memory for social events, Pers. Soc. Psychol. Bull. 26, 486 (2000).

[40] G. Downey and S. I. Feldman, Implications of rejection sensitivity for intimate relationships, J. Pers. Soc. Psychol. 70, 1327 (1996).

[41] C. L. Pickett, W. L. Gardner, and M. Knowles, Getting a cue: The need to belong and enhanced sensitivity to social cues, Pers. Soc. Psychol. Bull. 30, 1095 (2004).

[42] G. M. Walton and G. L. Cohen, A question of belonging: Race, social fit, and achievement, J. Pers. Soc. Psychol. 92, 82 (2007).

[43] 1. Aguilar, G. Walton, and C. Wieman, Psychological insights for improved physics teaching, Phys. Today 67, No. 5, 432014.

[44] N. Dasgupta, Ingroup experts and peers as social vaccines who inoculate the self-concept: The stereotype inoculation model, Psychol. Inq. 22, 231 (2011).

[45] J. G. Stout, N. Dasgupta, M. Hunsinger, and M. A. McManus, STEMing the tide: Using ingroup experts to inoculate women's self-concept in science, technology, engineering, and mathematics (STEM), J. Pers. Soc. Psychol. 100, 255 (2011).

[46] M. C. Murphy, C. M. Steele, and J. J. Gross, Signaling threat how situational cues affect women in Math, Science, and Engineering settings, Psychol. Sci. 18, 879 (2007).

[47] S. Cheryan, B. J. Drury, and M. Vichayapai, Enduring influence of stereotypical computer science role models on women's academic aspirations, Psychol. Women Q. 37, 72 (2013).

[48] S. Cheryan, V. C. Plaut, P. G. Davies, and C. M. Steele, Ambient belonging: How stereotypical cues impact gender participation in computer science, J. Pers. Soc. Psychol. 97, 1045 (2009).

[49] J. M. Chen and D. L. Hamilton, Understanding diversity the importance of social acceptance, Pers. Soc. Psychol. Bull. 41, 586 (2015).

[50] S. J. Spencer, C. M. Steele, and D. M. Quinn, Stereotype threat and women's math performance, J. Exp. Soc. Psychol. 35, 4 (1999).

[51] B. A. Nosek, M. R. Banaji, and A. G. Greenwald, Math = male, me $=$ female, therefore math $\neq$ me, J. Pers. Soc. Psychol. 83, 44 (2002).

[52] C. M. Steele, A threat in the air: How stereotypes shape intellectual identity and performance, Am. Psychol. 52, 613 (1997).

[53] D. Sekaquaptewa and M. Thompson, The differential effects of solo status on members of high- and low-status groups, Pers. Soc. Psychol. Bull. 28, 694 (2002).

[54] M. Inzlicht and T. Ben-Zeev, A threatening intellectual environment: Why females are susceptible to experiencing problem-solving deficits in the presence of males, Psychol. Sci. 11, 365 (2000).

[55] Dweck, Motivational processes affecting learning, Am. Psychol. 41, 1040 (1986).

[56] S. J. Leslie, A. Cimpian, M. Meyer, and E. Freeland, Expectations of brilliance underlie gender distributions across academic disciplines, Science 347, 262 (2015).
[57] J. L. Smith, K. L. Lewis, L. Hawthorne, and S. D. Hodges, When trying hard isn't natural women's belonging with and motivation for male-dominated stem fields as a function of effort expenditure concerns, Pers. Soc. Psychol. Bull. 39, 131 (2013).

[58] G. M. Walton and G. L. Cohen, A brief social-belonging intervention improves academic and health outcomes of minority students, Science 331, 1447 (2011).

[59] S. Cohen and T. A. Wills, Stress, social support, and the buffering hypothesis, Psychol. Bull. 98, 310 (1985).

[60] G. L. Cohen and D. K. Sherman, The psychology of change: Self-affirmation and social psychological intervention, Annu. Rev. Psychol. 65, 333 (2014).

[61] J. E. Cook, V. Purdie-Vaughns, J. Garcia, and G. L. Cohen, Chronic threat and contingent belonging: Protective benefits of values affirmation on identity development, J. Pers. Soc. Psychol. 102, 479 (2012).

[62] N. Schnabel, V. Purdie-Vaughns, J. E. Cook, J. Garcia, and G. L. Cohen, Demystifying values-affirmation interventions writing about social belonging is a key to buffering against identity threat, Pers. Soc. Psychol. Bull. 39, 663 (2013).

[63] G. L. Cohen, J. Garcia, N. Apfel, and A. Master, Reducing the racial achievement gap: A social-psychological intervention, Science 313, 1307 (2006).

[64] A. Miyake, L. E. Kost-Smith, N. D. Finkelstein, S. J. Pollock, G. L. Cohen, and T. A. Ito, Reducing the gender achievement gap in college science: A classroom study of values affirmation, Science 330, 1234 (2010).

[65] J. T. Cacioppo and B. Patrick, Loneliness: Human Nature and the Need for Social Connection (W. W. Norton \& Company, Inc., Norton, New York, 2008).

[66] L. McCullough and D.E. Meltzer, in 2001 Physics Education Research Conference Proceedings, Rochester, NY, 2001, edited by S. V. Franklin and K. Cummings, pp. 103-106.

[67] J. Trefil and S. Swartz, Problem sets and other deterrents for women, Phys. Today 65, No. 5, 10 (2012).

[68] E. Aronson and S. Patnoe, The Jigsaw Classroom. Building Cooperation in the Classroom (Addison-Wesley, MA, 1995).

[69] L. A. Meadows and D. Sekaquaptewa, in 2011 Proceedings of the Association for Engineering Education (ASEE) Annual Conference, Vancouver, BC, 2011.

[70] C. S. Hulleman, O. Godes, B. L. Hendricks, and J. M. Harackiewicz, Enhancing interest and performance with a utility value intervention, J. Educ. Psychol. 102, 880 (2010).

[71] D. B. Thoman, E. R. Brown, A. Z. Mason, A. G. Harmsen, and J. L. Smith, The role of altruistic values in motivating underrepresented minority students for biomedicine, BioScience 65, 183 (2015).

[72] L. Bowleg, When black + Lesbian + woman $\neq$ black lesbian woman: The methodological challenges of qualitative and quantitative intersectionality research, Sex Roles 59, 312 (2008).

[73] V. Purdie-Vaughns and R. P. Eibach, Intersectional invisibility: The distinctive advantages and disadvantages of multiple subordinate-group identities, Sex Roles 59, 377 (2008). 